\title{
40-Gb/s TDM-PON Downstream Link With Low-Cost EML Transmitter and APD-Based Electrical Duobinary Receiver
}

\author{
Bart Moeneclaey, Member, IEEE, Fabrice Blache, Joris Van Kerrebrouck, Student Member, IEEE, Romain Brenot, \\ Gertjan Coudyzer, Mohand Achouche, Xing-Zhi Qiu, Member, IEEE, Johan Bauwelinck, Member, IEEE and \\ Xin Yin, Member, IEEE
}

(Top-Scoring Paper)

\begin{abstract}
We report a cost-effective $40-\mathrm{Gb} / \mathrm{s}$ time-division multiplexing passive optical network (TDM-PON) downstream link using 3-level electrical duobinary (EDB) modulation. The transmitter consists of a compact electroabsorption-modulated laser (EML) module. The receiver contains an avalanche photodiode (APD) packaged with a transimpedance amplifier (TIA), with digitally adjustable gain and bandwidth, followed by a duobinary decoder. Real-time eye diagram and bit-error rate (BER) measurements were performed. At a pre-forward error correction (FEC) BER of $10^{-3}$, a power budget of $23.6 \mathrm{~dB}$ in back-to-back conditions is demonstrated. For a dispersion range of $-215 \mathrm{ps} / \mathrm{nm}$ to $128 \mathrm{ps} / \mathrm{nm}$, the power penalty does not exceed $3 \mathrm{~dB}$.
\end{abstract}

Index Terms-passive optical network, TDM-PON, electrical duobinary

\section{INTRODUCTION}

$\mathbf{T}$ HE Full Service Access Network group [1] has decided that Next-Generation Passive Optical Network 2 (NGPON2) will be a time- and wavelength-division multiplexing passive optical network (TWDM-PON) wherein four or eight $10-\mathrm{Gb} / \mathrm{s}$ streams are stacked at different wavelengths [2]. The choice for abandoning the single-wavelength evolutionary path in favor of TWDM-PON was motivated by the foreseen technology limitations at that time; higher serial rate timedivision multiplexing passive optical networks (TDM-PONs) were deemed not cost-effective [3].

However, as the demand for broadband services continued to rise, the research community has demonstrated the feasibility of serial rates beyond $10 \mathrm{~Gb} / \mathrm{s}$, showing its potential

Manuscript received XXX XX, 201X; revised XXX XX, 201X; accepted XXX XX, 201X. Date of publication XXX XX, 201X; date of current version XXX XX, 201X.

This work was supported by the EU-funded FP7 ICT project DISCUS. The work of B. Moeneclaey was supported by a grant from the Institute for the Promotion of Innovation by Science and Technology (IWT) in Flanders.

B. Moeneclaey, J. Van Kerrebrouck, G. Coudyzer, X-Z. Qiu, J. Bauwelinck, and $\mathrm{X}$. Yin are with INTEC/IMEC- iMinds, Ghent University, Technologiepark-Zwijnaarde 15, 9052 Ghent, Belgium. (email: bart.moeneclaey@intec.ugent.be; joris.vankerrebrouck@intec.ugent.be; gertjan.coudyzer@intec.ugent.be;

johan.bauwelinck@intec.ugent.be; xin.yin@intec.ugent.be).

F. Blache, R. Brenot, and M. Achouche are with III- V Lab, Route de Nozay, 91460 Marcoussis, France. (e-mail: fabrice.blache@3-5lab.fr; romain.brenot@3-5lab.fr; mohand.achouche@3-5lab.fr).

Color versions of one or more of the figures in this letter are available online at http://ieeexplore.ieee.org. Digital Object Identifier XX.XXXX/JLT.201X.XXXXXX as a per-wavelength upgrade path for NG-PON2 [3]-[11]. Furthermore, the IEEE has not yet settled on the architecture for Next Generation Ethernet Passive Optical Network (NGEPON); both single-wavelength TDM-PON and TWDM-PON are still under consideration as viable candidates [12], [13].

The main challenges to overcome when increasing the serial data rate beyond $10 \mathrm{~Gb} / \mathrm{s}$ are decreased tolerance to chromatic dispersion (CD) and increased bandwidth requirements on the optical and electrical components, resulting in a reduced optical power budget, fiber reach and higher overall system cost [14]. These challenges are adressed by advanced modulation formats beyond non-return-to-zero (NRZ); electrical duobinary (EDB), optical duobinary (ODB) and 4-level pulse-amplitude modulation (PAM-4). In the case of EDB, NRZ is electrically low-pass filtered by the transmitter and/or receiver yielding a 3-level signal at the receiver output. ODB, on the other hand, requires modulating both optical amplitude and phase by means of a Mach-Zehnder Modulator (MZM), giving rise to an NRZ signal at the photodetector output. EDB, ODB, and PAM- 4 are strong candidates for high serial rate passive optical networks due to their relative simplicity and improved CD tolerance with respect to NRZ. Furthermore, EDB and PAM-4 permit the use of low-bandwidth components [13].

We already demonstrated EDB detection in a $25 \mathrm{~Gb} / \mathrm{s}$ TDMPON upstream link using a low-cost burst-mode receiver [15] at the optical line terminal (OLT). This was later extended in [8], which also demonstrated 50-Gb/s EDB transmission in the downstream direction. The OLT transmitter was comprised of a MZM whose output power was boosted using a semiconductor optical amplifier (SOA), whereas the optical network unit (ONU) receiver consisted of a receiver based on a SOA-preamplified PIN photodiode, followed by a duobinary decoder.

This paper is an invited extension of our work presented in [7], wherein we presented a cost-effective $40-\mathrm{Gb} / \mathrm{s}$ singlewavelength TDM-PON downstream utilizing high-power DFB laser integrated with an EAM, henceforth denoted as DFBEAM, in the OLT and an EDB receiver with an APD in the ONU. Real-time measurements were performed in the C-band where a loss budget margin of $20.7 \mathrm{~dB}$ has been achieved at $40 \mathrm{~Gb} / \mathrm{s}$ in the measured dispersion range of $-215 \mathrm{ps} / \mathrm{nm}$ to $128 \mathrm{ps} / \mathrm{nm}$. To the best of our knowledge, this was the first time a $40-\mathrm{Gb} / \mathrm{s}$ TDM-PON downstream link was achieved in 


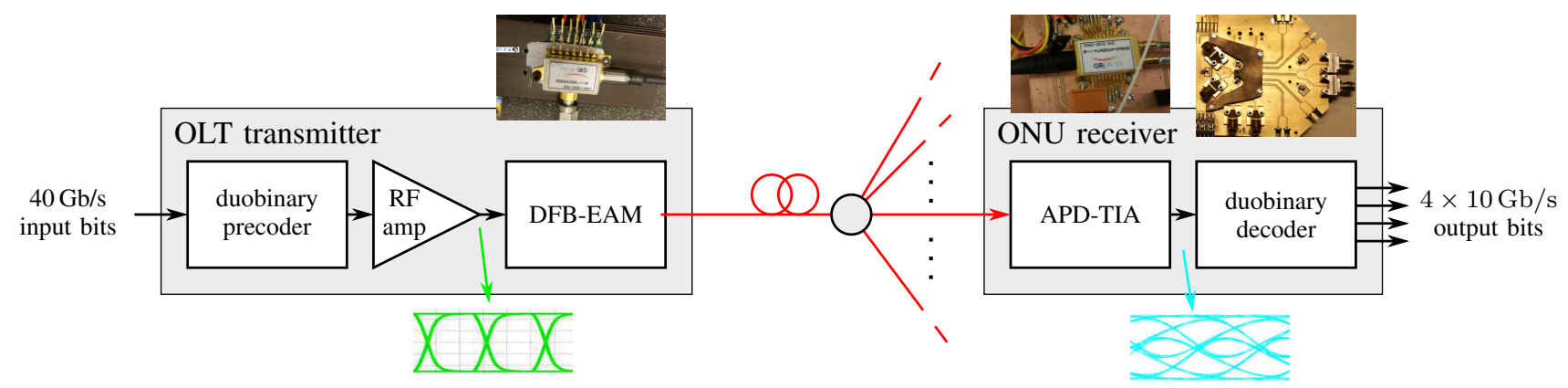

Fig. 1. A cost-effective implementation of a 40-Gb/s TDM-PON downstream link using EDB modulation: utilizing an integrated high-power DFB-EAM transmitter in the OLT and an APD-based 3-level detection receiver in the ONU.

real-time experiments utilizing low-cost components at both OLT and ONU, without any offline digital signal processing. In the present paper we report more details on the ONU receiver through additional measurements and simulations.

Section II presents the investigated low-cost $40 \mathrm{~Gb} / \mathrm{s}$ downstream link and discusses the transmitter and receiver prototypes. The experimental setup and the results of bit-error rate (BER) and eye diagram measurements are presented in Section III.

\section{LOW-COST $40 \mathrm{~GB} / \mathrm{S}$ DOWNSTREAM TRANSMITTER AND RECEIVER PROTOTYPES}

The investigated low-cost $40 \mathrm{~Gb} / \mathrm{s}$ EDB downstream link is illustrated in Fig. 1. At the OLT, a compact and low-cost integrated DFB-EAM transmitter module is modulated by an on-off keying NRZ signal. Usage of EDB requires that the cascade of the transmitter electro-optical response $H_{\mathrm{TX}}(f)$ and receiver opto-electrical response $H_{\mathrm{RX}}(f)$ forms a baseband cosine filter, i.e.,

$$
\left|H_{\mathrm{TX}}(f) \cdot H_{\mathrm{RX}}(f)\right|= \begin{cases}\cos (\pi f T), & |f| \leq 1 /(2 T) \\ 0, & |f|>1 /(2 T)\end{cases}
$$

where $T$ denotes the bit period [16]. Note that the $3-\mathrm{dB}$ bandwidth of this filter is $1 /(4 T)$, which allows lower-bandwidth optoelectronic components (e.g., electroabsorption-modulated laser (EML) in the OLT and APD employed at the ONU), reducing the cost and power consumption compared to NRZ or ODB. The cosine filter response is approximated by the use of lower-bandwidth optoelectronic components, along with appropriate bandwidth selection of the transimpedance amplifier (TIA) input stage, as will be discussed in Section II-B. As such, the use of equalization in either OLT or ONU, and the corresponding increase in cost and power consumption, is avoided. Simulations indicate that the reduced bandwidth can further improve the $\mathrm{CD}$ tolerance and receiver sensitivity in high serial data rate downstream applications [17]. In the ONU, the intensity modulated signal enters the receiver and is converted into a 3-level EDB signal with levels $-V, 0$, and $+V$. To avoid error propagation, a precoder is placed at the transmitter which implements the function $y_{k}=x_{k} \oplus y_{k-1}$, where $x_{k}$ is the $k$-th uncoded bit, $y_{k}$ is the $k$-th precoded bit, and $\oplus$ represents modulo-2 addition [16]. The precoder allows the duobinary decoder in the receiver to use symbol-by-symbol

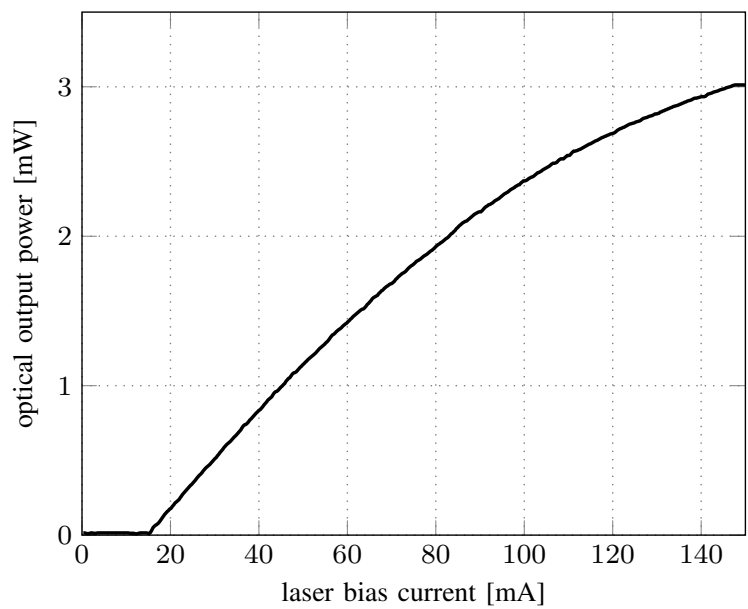

Fig. 2. EML optical output power versus laser bias current at $45^{\circ} \mathrm{C}$ with EAM unbiased.

decision [16]; the levels $\pm V$ correspond to a 1-bit whereas the level 0 corresponds to a 0-bit.

\section{A. OLT transmitter}

The transmitter module is an EML based on an InGaAsP DFB-EAM fabricated using the butt-joint approach, where the EAM is obtained by epitaxial regrowth and the DFB and EAM structures can be independently optimized [18]. The device was optimized for high output power at $45^{\circ} \mathrm{C}$, resulting in a low power consumption of the thermoelectric cooler. The output power is an important metric of the laser as it needs to overcome the insertion loss of the EAM, while fulfilling the power budget requirements.

Fig. 2 shows the optical output power of the EML versus the laser bias current, where the EAM was left unbiased and the temperature was regulated to $45^{\circ} \mathrm{C}$. The optical power at the fiber output of the EML reaches $2.4 \mathrm{~mW}$ at a $100-\mathrm{mA}$ drive current and can reach $3 \mathrm{~mW}$ when the current is increased to $145 \mathrm{~mA}$. The relative transmission curves of the EAM are shown in Fig. 3 for various DFB laser currents. The static extinction ratio between 0 and $-3 \mathrm{~V}$ is approximately $14 \mathrm{~dB}$, with a modulation slope of $10 \mathrm{~dB} / \mathrm{V}$.

The whole DFB-EAM chip was integrated on a submount and installed in a butterfly package. The electro-optical frequency response and return loss of the packaged EML were 


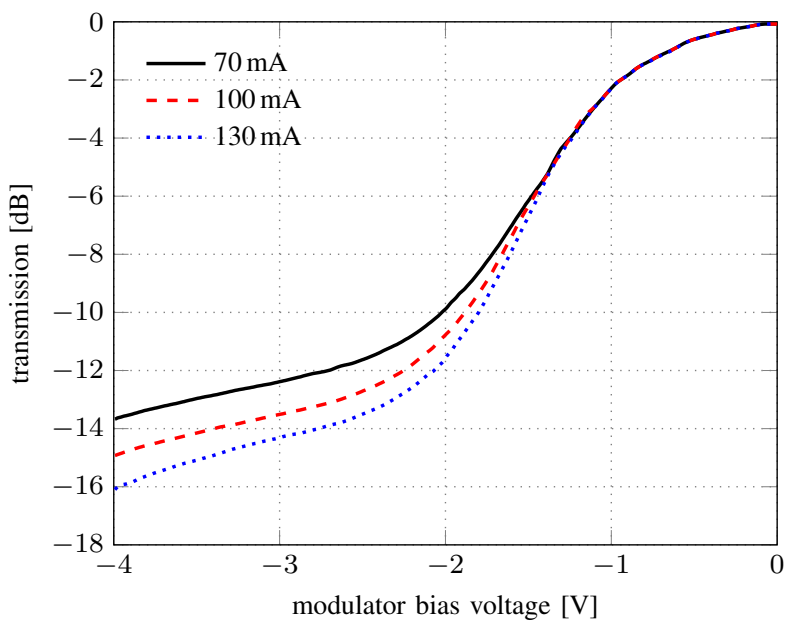

Fig. 3. Transmission curves of the EML for various laser currents.

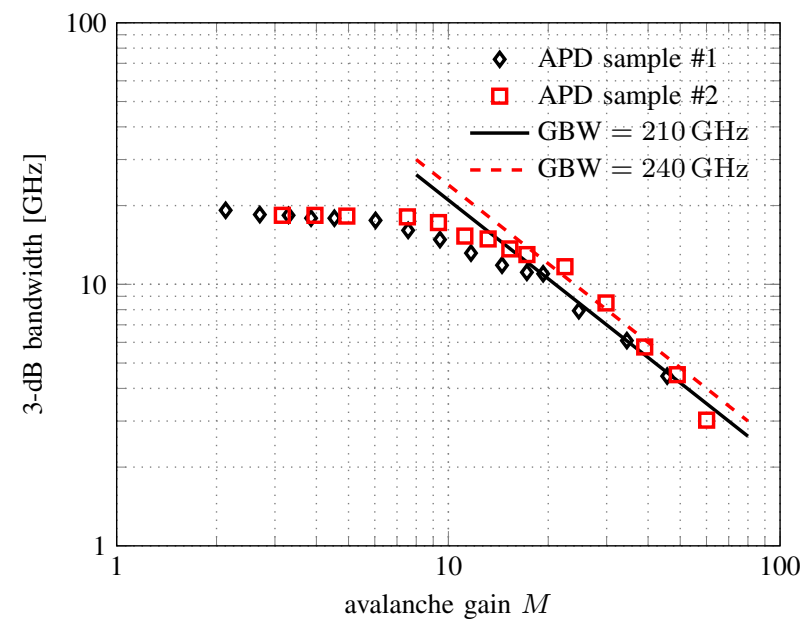

Fig. 4. APD 3-dB bandwidth versus avalanche gain $M$.

measured at an operating temperature of $45^{\circ} \mathrm{C}$, a DFB laser current of $70 \mathrm{~mA}$, and an EAM bias voltage of $-1.3 \mathrm{~V}$. The normalized electro-optical frequency response has a 3-dB bandwidth exceeding $20 \mathrm{GHz}$, whereas the return loss remains lower than $-10 \mathrm{~dB}$ up to $20 \mathrm{GHz}$.

\section{B. ONU receiver}

The ONU receiver is comprised of a linear APD-based TIA, installed in a butterfly package, followed by a 3-level EDB decoder. Due to the nature of the investigated 3-level EDB modulation scheme, a linear TIA front-end is required to preserve vertical eye openings, while providing sufficient gain to ease further signal handling in the subsequent decoder.

The photodiode is a back-side illuminated AlInAs/GaInAs APD fabricated using a highly reliable planar junction process carried out by Zn diffusion. Fig. 4 shows the high-frequency response of the APD versus avalanche gain $M$, while the avalanche gain $M$ is plotted versus the APD reverse voltage $V_{\mathrm{APD}}$ in Fig. 5. A 3-dB bandwidth between $18 \mathrm{GHz}$ and $20 \mathrm{GHz}$ is achieved for avalanche gains between 2 and 8 , and the extrapolated gain-bandwidth product is about $220 \mathrm{GHz}$.

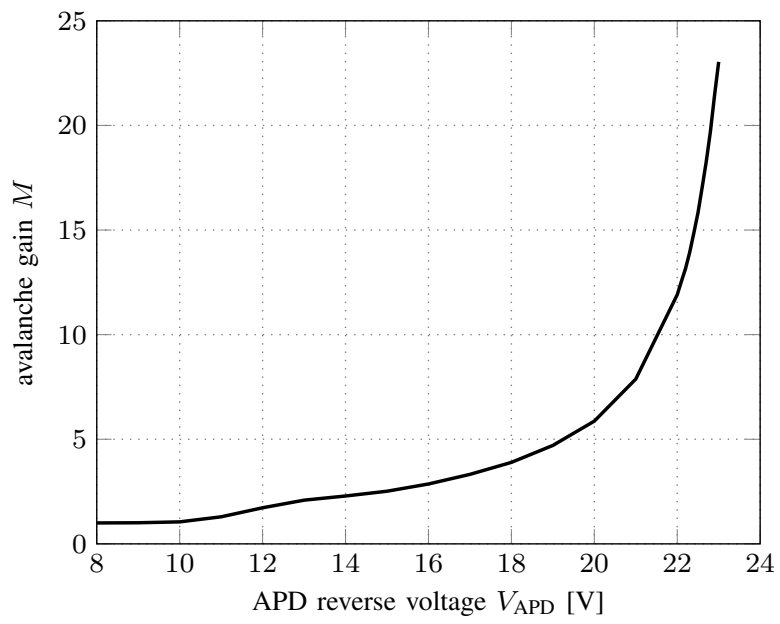

Fig. 5. APD avalanche gain $M$ vs APD reverse voltage $V_{\mathrm{APD}}$.

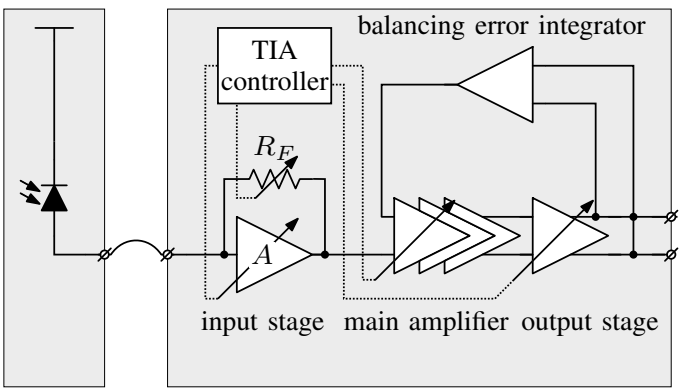

Fig. 6. TIA architecture block diagram.

Fig. 6 shows a simplified block diagram of the TIA. The datapath consists of a single-ended shunt-feedback TIA input stage, followed by a differential main amplifier and output stage. The balancing error integrator closes a feedback loop which removes the dc-offset between both output signals by adjusting the dc-voltage at the inverting input of the main amplifier. The built-in controller allows to digitally vary the gain and associated linearity of the data path, as well as its $3-\mathrm{dB}$ bandwidth. The transimpedance gain and bandwidth of the input stage can be set by modifying the feedback resistor $R_{F}$ and the amplifier gain $A$. This is discussed in detail below. Furthermore, the gain of the main amplifier and output stage can be changed as well by modifying the emitter degeneration in each stage, such that the output signal swing can be kept at a reasonable level for varying optical input powers. The TIA runs off a $2.5 \mathrm{~V}$ supply and consumes $150 \mathrm{~mW}$. The chip was manufactured in a $0.13 \mu \mathrm{m}$ SiGe BiCMOS process and its die micrograph is shown in Fig. 7. The total chip area is $2200 \mu \mathrm{m} \times 1000 \mu \mathrm{m}$.

Fig. 8 shows a simplified circuit diagram of the TIA input stage. The design is a shunt-shunt feedback amplifier, based on [19], and features a variable feedback resistor $R_{F}$ and emitter degeneration resistor $R_{E}$ in order to manipulate the transimpedance gain as well as the bandwidth of the input stage. This enables optimization of the TIA for either NRZ or EDB reception. Both variable resistors are implemented as nchannel metal-oxide-semiconductor transistor arrays biased in 


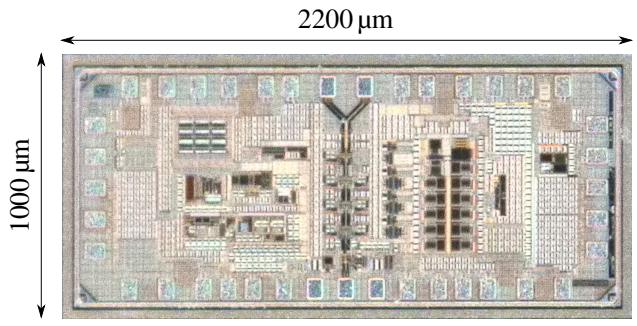

Fig. 7. Die micrograph of the TIA.

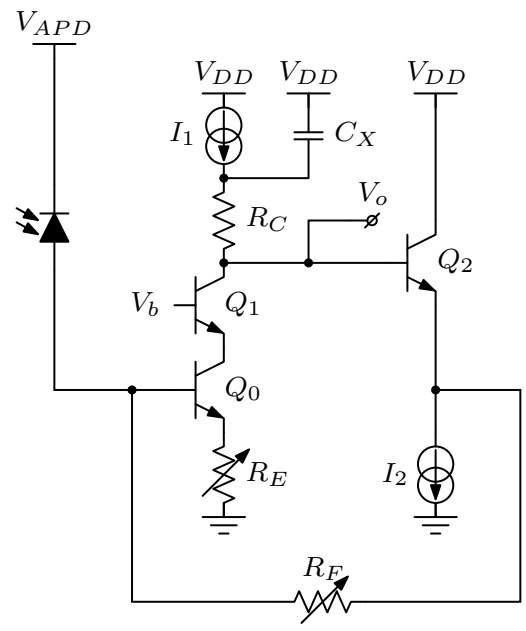

Fig. 8. Simplified circuit diagram of the transimpedance input stage.

the linear region. As such, they can be controlled digitally by selectively enabling individual transistors: $R_{F}=1.1 \mathrm{k} \Omega / N$ with $N=1,2, \ldots 16$ and $R_{E}=24 \Omega / M$ with $M=$ $1,2, \ldots 16$. The forward path consists of a common-emitter amplifier where $Q_{0}$ is degenerated by $R_{E}$ which controls the voltage gain of said amplifier. Current source $I_{1}$ provides a supply-independent bias current to the amplifier and is decoupled by capacitor $C_{X}$ which is dimensioned such that the current source is effectively short-circuited for the frequencies of interest [19].

The transimpedance gain is in large part controlled by the feedback resistor $R_{F}$. However, $R_{F}$ also affects the dominant pole in the loop gain, which can be counteracted by modifying the voltage gain of the common-emitter amplifier by means of $R_{E}$. As the dominant pole is increased and approaches the non-dominant poles, the phase margin is reduced, the closed loop transfer function exhibits peaking and can eventually become unstable. This is illustrated in Fig. 9 which shows a contour plot of the transimpedance gain and 3-dB bandwidth, obtained using $\mathrm{AC}$ analysis simulations on the input stage. The portion of the $\left(R_{F}, R_{E}\right)$ space for which the peaking was larger than $1 \mathrm{~dB}$ is also annotated in the figure. As indicated by Fig. 9, the TIA input stage supports a wide range of transimpedance gains $(150 \Omega$ to $300 \Omega$ for a $20 \mathrm{GHz} 3-\mathrm{dB}$ bandwidth) and $3-\mathrm{dB}$ bandwidths (wider than the range from $10 \mathrm{GHz}$ to $30 \mathrm{GHz}$ for a $200 \Omega$ transimpedance gain).

The EDB decoder [20] combines a 3-level signal decoding function and a $1 \div 4$ deserializer. The TIA output signal is compared to two configurable threshold levels. The two comparator outputs are then processed by a high-speed XOR gate

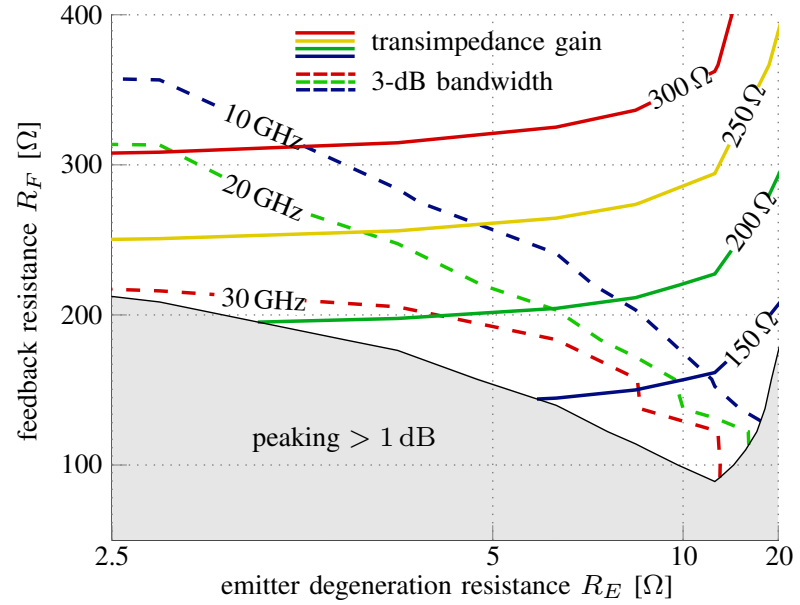

Fig. 9. Contour plot of the TIA input stage transimpedance and 3-dB bandwidth versus feedback resistance $R_{F}$ and emitter degeneration resistance $R_{E}$.

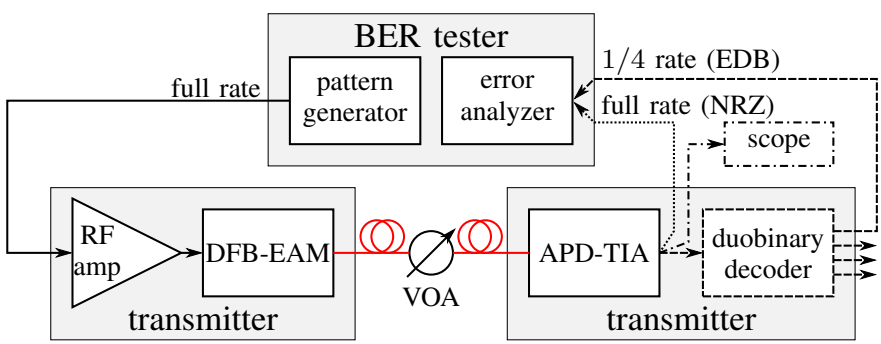

Fig. 10. Experimental setup for BER and eye diagram measurements. For EDB BER measurements (dashed lines), the differential TIA outputs are connected to the duobinary decoder, of which one $1 / 4$ rate output is connected to the error analyzer. NRZ BER measurements (dotted lines) directly connect the differential TIA outputs to the error analyzer. For eye diagram measurements (dash-dot lines), the differential TIA outputs are directly connected to an oscilloscope.

and deserialized into 4 outputs for interfacing with lower-speed off-chip components, e.g., field-programmable gate arrays.

\section{EXPERIMENTAL SETUP AND RESULTS}

In this section, we present BER and eye diagram measurement results for both EDB and NRZ modulation formats. Figure 10 depicts the experimental setup used for these measurements. The transmitter was driven by a $2^{7}-1$ pseudorandom binary sequence (PRBS) full-rate NRZ signal originating from a pattern generator. The DFB laser current was set to $150 \mathrm{~mA}$, whereas the EAM was biased with a voltage of $-1.55 \mathrm{~V}$. With a thermoelectric cooler regulating the EML temperature at $25^{\circ} \mathrm{C}$, the average transmitted optical output power was $4 \mathrm{dBm}$ at a central wavelength of $1557 \mathrm{~nm}$. The extinction ratio was approximately $13 \mathrm{~dB}$. The modulated light passes through a variable optical attenuator and finally reaches the APD through the pigtail connector on the receiver module. The APD was biased with a reverse voltage of $V_{A P D}=21 \mathrm{~V}$. For EDB BER measurements, the differential TIA outputs were connected to the duobinary decoder, which fed one of its four demultiplexed 1/4-rate outputs to the error analyzer. For NRZ BER measurements, on the other hand, the duobinary decoder was omitted and the differential TIA outputs were 


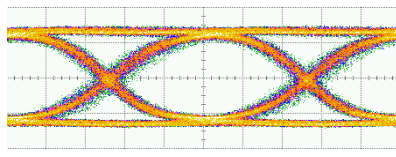

(a) $20 \mathrm{~Gb} / \mathrm{s} \mathrm{NRZ}$

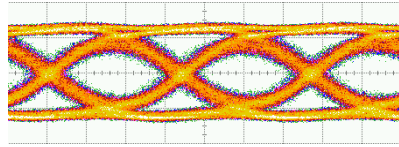

(c) $30 \mathrm{~Gb} / \mathrm{s} \mathrm{NRZ}$ (b) $25 \mathrm{~Gb} / \mathrm{s} \mathrm{NRZ}$

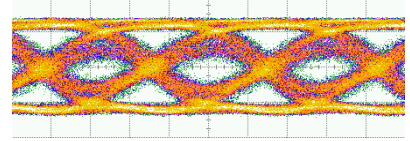

(d) $35 \mathrm{~Gb} / \mathrm{s}$ NRZ

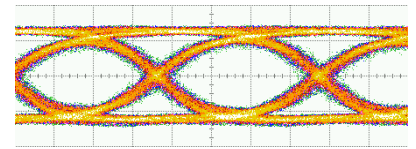

Fig. 11. Measured eye diagrams of the differential TIA output voltage in backto-back conditions for (a) $20 \mathrm{~Gb} / \mathrm{s}$, (b) $25 \mathrm{~Gb} / \mathrm{s}$, (c) $30 \mathrm{~Gb} / \mathrm{s}$, and (d) $35 \mathrm{~Gb} / \mathrm{s}$ NRZ.

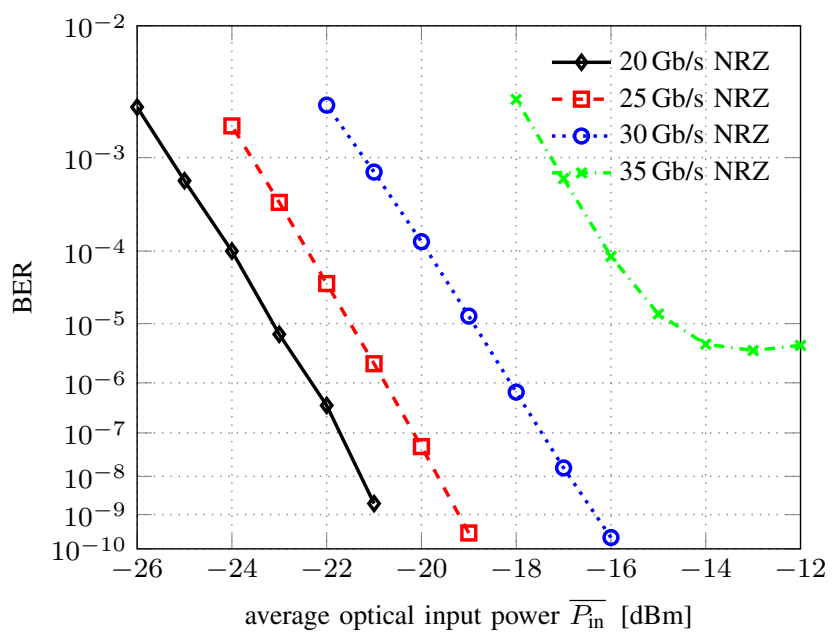

Fig. 12. Measured NRZ BER curves in back-to-back conditions for $20 \mathrm{~Gb} / \mathrm{s}$, $25 \mathrm{~Gb} / \mathrm{s}, 30 \mathrm{~Gb} / \mathrm{s}$ and $35 \mathrm{~Gb} / \mathrm{s}$.

directly connected to error analyzer. Finally, eye diagrams were measured by connecting the differential TIA outputs directly to an oscilloscope. To compare the performance of NRZ and EDB, the TIA settings were optimized for the corresponding modulation scheme. Note from Fig. 10 that the duobinary precoder is omitted from the experimental setup, not only when using the NRZ modulation format, but also in the case of EDB; this is because the precoder operating on the PRBS signal yields a delayed version of the PRBS signal [20]. As such, the precoder can be simply removed when using PRBS data [4]-[8], [20].

Fig. 11 shows the eye diagrams obtained for NRZ signaling rates from $20 \mathrm{~Gb} / \mathrm{s}$ to $35 \mathrm{~Gb} / \mathrm{s}$, whereas Fig. 12 presents the corresponding BERs, measured at the TIA output, versus the average received optical power. The NRZ eye diagrams for bit rates up to $30 \mathrm{~Gb} / \mathrm{s}$ show sufficient horizontal and vertical eye opening. For $35 \mathrm{~Gb} / \mathrm{s}$, however, the bandwidth of the receiver is insufficient, resulting in considerable intersymbol interference reducing the eye opening. This is also observed in the $35 \mathrm{~Gb} / \mathrm{s}$ BER curve in Fig. 12, showing a deteriorated sensitivity of $-17.3 \mathrm{dBm}$ at the pre-forward error correction (FEC) BER threshold of $10^{-3}$ as well as a BER floor slightly below $10^{-5}$.

Fig. 13 shows the measured $40 \mathrm{~Gb} / \mathrm{s}$ 3-level EDB eye diagram of the differential TIA output voltage, exhibiting

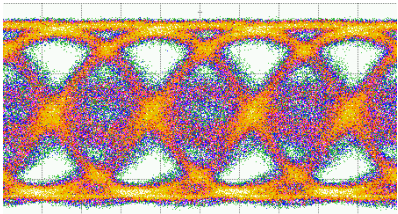

Fig. 13. Measured eye diagram of the differential TIA output voltage in back-to-back conditions for $40 \mathrm{~Gb} / \mathrm{s}$ EDB.

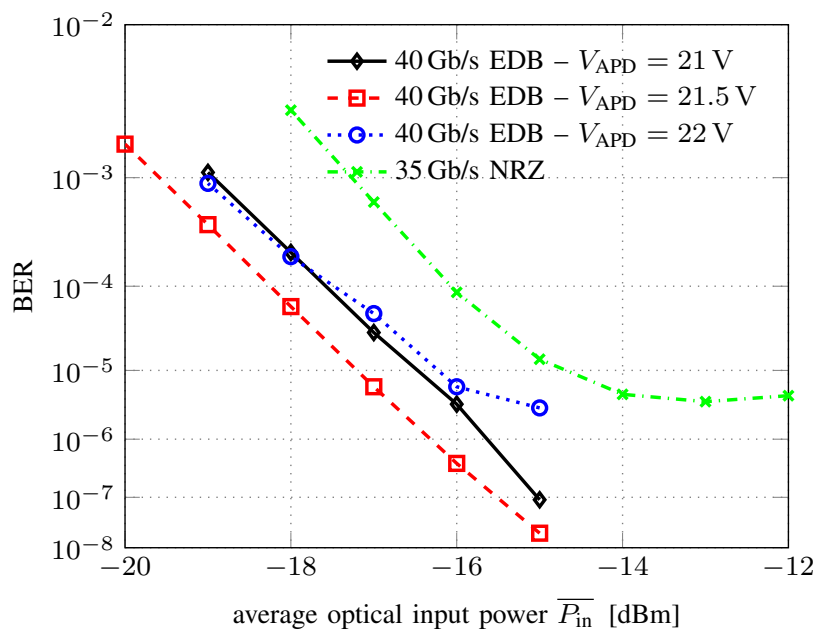

Fig. 14. Measured $40 \mathrm{~Gb} / \mathrm{s}$ EDB BER curves in back-to-back conditions for different APD voltages.

sufficient opening in both eyes. The effect of the APD reverse voltage $V_{A P D}$ and consequently the avalanche gain $M$ was investigated by measuring the BER curves for $V_{A P D}=$ $21 \mathrm{~V}, 21.5 \mathrm{~V}$ and $22 \mathrm{~V}$. These curves, along with the BER curve for $35 \mathrm{~Gb} / \mathrm{s} \mathrm{NRZ}$ are shown in Fig. 14. The best EDB sensitivity is obtained for $V_{A P D}=21.5 \mathrm{~V}$, yielding a $\mathrm{BER}=10^{-3}$ pre-FEC sensitivity of $-19.6 \mathrm{dBm}$; this constitutes a $2.3 \mathrm{~dB}$ improvement with respect to $35 \mathrm{~Gb} / \mathrm{s}$ NRZ transmission. Further increasing the APD bias voltage reduces the receiver bandwidth, introducing more intersymbol interference resulting in a deteriorated sensitivity. With the EDB sensitivity at $-19.6 \mathrm{dBm}$ and the transmitter average launched output power at $4 \mathrm{dBm}$, the power budget equals $23.6 \mathrm{~dB}$ in back-to-back conditions.

Next, we have evaluated the $40 \mathrm{~Gb} / \mathrm{s}$ 3-level EDB link as a function of dispersion. The measured power penalties versus various dispersion values are shown in Fig. 15 together with simulated results for $40 \mathrm{~Gb} / \mathrm{s} \mathrm{NRZ} \mathrm{and} \mathrm{EDB} \mathrm{obtained}$ in [17]. The positive dispersion points were measured with various lengths of standard single-mode fiber (SSMF), while the negative dispersion points were measured with a tunable dispersion emulator. We also cross-checked the results of positive dispersion using both the SSMF and the dispersion emulator, and both measurements gave comparable power penalty. The resulting maximal power penalty compared to back-toback conditions was $2.9 \mathrm{~dB}$ in the range from $-215 \mathrm{ps} / \mathrm{nm}$ to $128 \mathrm{ps} / \mathrm{nm}$. Hence, with appropriate dispersion compensation, the proposed EDB TDM-PON downstream link supports a differential reach of $20 \mathrm{~km}$ SSMF (assuming a dispersion value of $17 \mathrm{ps} /(\mathrm{nm} \mathrm{km}))$ and a $20.7 \mathrm{~dB}$ power budget. As such, it meets 


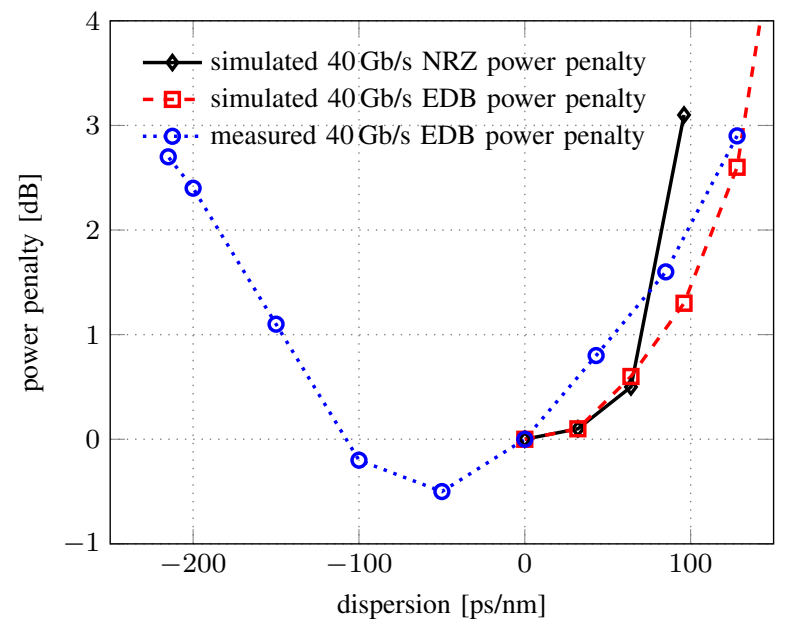

Fig. 15. Measured $40 \mathrm{~Gb} / \mathrm{s}$ EDB power penalty due to chromatic dispersion compared with simulation results for $40 \mathrm{~Gb} / \mathrm{s}$ NRZ and EDB.

the legacy power budget and differential reach requirements of Gigabit-capable Passive Optical Network (GPON) class A [21] and $10 \mathrm{~Gb} / \mathrm{s}$ Ethernet Passive Optical Network (10G-EPON) classes PRX10 and PR10 [22].

\section{CONCLUSIONS}

We have investigated a low-cost $40 \mathrm{~Gb} / \mathrm{s}$ TDM-PON downstream link using EDB modulation. At the OLT, the transmitter is comprised of a DFB-EAM modulated by an NRZ signal. The ONU receiver consists of an APD-based TIA followed by a 3-level duobinary decoder. The TIA transimpedance gain and associated linearity as well as its bandwidth can be digitally varied, enabling optimization for either NRZ or EDB reception. For the first time, real-time measurements using $40 \mathrm{~Gb} / \mathrm{s}$ downstream EDB transmission were performed with compact and low-cost components in both the OLT and ONU. A high power budget of $23.6 \mathrm{~dB}$ has been achieved in back-toback conditions. The measured power penalty remained below $3 \mathrm{~dB}$ for the dispersion range from $-215 \mathrm{ps} / \mathrm{nm}$ to $128 \mathrm{ps} / \mathrm{nm}$.

\section{REFERENCES}

[1] Full Service Access Network (FSAN), 2016. [Online]. Available: http://www.fsan.org/

[2] ITU-T G.989.1, "40-Gigabit-capable passive optical networks (NGPON2): General requirements," 2013.

[3] V. Houtsma and D. van Veen, "Demonstration of symmetrical 25 Gbps TDM-PON with $31.5 \mathrm{~dB}$ optical power budget using only $10 \mathrm{Gbps}$ optical components," in Eur. Conf. Opt. Commun., 2015.

[4] V. Houtsma, D. van Veen, A. Gnauck, and P. Iannone, "APD-Based DuoBinary Direct Detection Receivers for 40 Gbps TDM-PON," in Opt. Fiber Commun. Conf., 2015, p. Th4H.1.

[5] D. T. van Veen and V. E. Houtsma, "Symmetrical 25-Gb/s TDM-PON With 31.5-dB Optical Power Budget Using Only Off-the-Shelf 10-Gb/s Optical Components," J. Lightw. Technol., vol. 34, no. 7, pp. 1636-1642, apr 2016.

[6] D. T. Van Veen, V. E. Houtsma, A. H. Gnauck, and P. Iannone, "Demonstration of 40-Gb/s TDM-PON Over 42-km With $31 \mathrm{~dB}$ Optical Power Budget Using an APD-Based Receiver," J. Lightw. Technol., vol. 33, no. 8, pp. 1675-1680, apr 2015.

[7] X. Yin et al., "40-Gb/s TDM-PON Downstream with Low-Cost EML Transmitter and 3-Level Detection APD Receiver," in Opt. Fiber Commun. Conf., 2016.
[8] _ "An Asymmetric High Serial Rate TDM-PON With Single Carrier $25 \mathrm{~Gb} / \mathrm{s}$ Upstream and $50 \mathrm{~Gb} / \mathrm{s}$ Downstream," J. Lightw. Technol. vol. 34, no. 2, pp. 819-825, jan 2016.

[9] Z. Zhou, M. Bi, S. Xiao, Y. Zhang, and W. Hu, "Experimental Demonstration of Symmetric 100-Gb/s DML-Based TWDM-PON System," IEEE Photon. Technol. Lett., vol. 27, no. 5, pp. 470-473, mar 2015.

[10] H. Zhang, S. Fu, J. Man, W. Chen, X. Song, and L. Zeng, "30km Downstream Transmission Using 425Gb/s 4-PAM Modulation with Commercial 10Gbps TOSA and ROSA for 100Gb/s-PON," in Opt. Fiber Commun. Conf., 2014.

[11] Z. Ye, S. Li, N. Cheng, and X. Liu, "Demonstration of high-performance cost-effective 100-Gb/s TWDM-PON using 4x25-Gb/s optical duobinary channels with 16-GHz APD and receiver-side post-equalization," in Eur. Conf. Opt. Commun., sep 2015.

[12] IEEE 802.3 Ethernet Working Group Communication, "IEEE 802.3 Industry Connections Feasibility Assessment for the Next Generation of EPON," 2015. [Online]. Available: http://www.ieee802.org/3/ad_hoc/ngepon/ng_epon_report.pdf

[13] P. P. Iannone, A. H. Gnauck, D. T. van Veen, and V. E. Houtsma, "Increasing TDM Rates for Access Systems Beyond NG-PON2," $J$. Lightw. Technol., vol. 34, no. 6, pp. 1545-1550, mar 2016.

[14] D. Van Veen and V. Houtsma, "High speed TDM PON beyond 10G," in Opt. Fiber Commun. Conf., 2016.

[15] X. Yin et al., "25Gb/s 3-level burst-mode receiver for high serial rate TDM-PONs," in Opt. Fiber Commun. Conf., 2015.

[16] I. A. Glover and P. M. Grant, Digital communications. Pearson Education, 2010.

[17] X. Yin et al., "Performance evaluation of single carrier 40-Gbit/s downstream for long-reach passive optical networks," in Int. Conf. Opt. Network Design and Modeling, 2014.

[18] G. Ghione, Semiconductor Devices for High-speed Optoelectronics. Cambridge University Press, 2009.

[19] B. Moeneclaey et al., "A 40-Gb/s Transimpedance Amplifier for Optical Links," IEEE Photon. Technol. Lett., vol. 27, no. 13, pp. 1375-1378, jul 2015.

[20] T. De Keulenaer et al., "84 Gbit/s SiGe BiCMOS duobinary serial data link including Serialiser/Deserialiser (SERDES) and 5-tap FFE," Electronics Letters, vol. 51, no. 4, pp. 343-345, feb 2015.

[21] ITU-T G.984.2, "Gigabit-capable Passive Optical Networks (GPON): Physical Media Dependent (PMD) layer specification," 2003.

[22] IEEE Std 802.3av-2009, "IEEE Standard for Information technologyLocal and metropolitan area networks- Specific requirements- Part 3: CSMA/CD Access Method and Physical Layer Specifications Amendment 1: Physical Layer Specifications and Management Parameters for 10 Gb/s Passive Optical Networks," 2009.

Bart Moeneclaey (M'14) was born in Ghent, Belgium, in 1988. He received the engineering degree in applied electronics from Ghent University, Ghent, Belgium, in 2011 where he is currently working toward the Ph.D. degree. He has been a Research Assistant in the INTEC_design laboratory, Ghent University, since 2011. His research is focused on amplifier circuit design for high-speed optical communication systems.

Fabrice Blache received the Ph.D. degree in electronics from the University of Limoges, Limoges, France, in 1995. He is currently a Research Engineer with the III-V Lab, Marcoussis, France, a joint laboratory of Alcatel-Lucent Bell Labs France, Thales Research \& Technology, and CEA-Leti, where his main expertises are in the field of high-frequency designs for optoelectronic modules. He is currently leading research activities for $100-\mathrm{Gb} / \mathrm{s}$ applications.

Joris Van Kerrebrouck was born in Ghent, Belgium in 1989. He received the master degree in applied electrical engineering from Ghent University, Belgium, in 2014. In that year, he joined the INTEC Design laboratory part of the department of information technology at Ghent University, where he pursues the Ph.D. degree, working on high speed electrical transceivers. His current fields of interest are high-speed SiGe BiCMOS analog circuits. 
Romain Brenot was born in LHay-Les-Roses, France, in 1972. He entered Ecole Normale Superieure, Paris, France in 1992. He received the Ph.D. degree in solid state physics from Ecole Poly-technique, Palaiseau, France in 2000 for works on transport properties of microcrystalline silicon. Since 2000, he has been with Alcatel Research and Innovation, Alcatel-Thales III-V Laboratories, Marcoussis Cedex, France, and has been involved in designing InP-based opto- electronic devices.

Gertjan Coudyzer was born in 1990 in Ronse, Belgium. After receiving the master degree in electrical engineering, he joined the INTEC Design laboratory as part of the department of information technology at Ghent University. There, he is currently working towards the Ph.D. degree in electrical engineering. His research is focused on the development of highspeed continuous and burst-mode electrical receivers.

Mohand Achouche received the Ph.D. degree from Paris Diderot University (Jussieu), Paris, France, in 1996. His research activities started in 1993 at the National Centre of Telecommunication Research (CNET, Laboratoire de Bagneux) on the fabrication and characterization of high-electron-mobility transistor tran- sistors for high-speed circuits. During 1997-2000, he was with Ferdinand Braun Institute (FBH-Berlin) working on power amplifiers based on $\mathrm{GaAs}$ heterojunction bipolar transistors for mobile communications. In 2000, he joined Alcatel Research and Innovation, where he was first involved in $40 \mathrm{~Gb} / \mathrm{s}$ photodiodes. In 2004, he was in charge of a research team on photoreceivers for high-speed optical communication systems, microwave photonics links, and sensors. In 2011, he was in charge of Photonic integrated Circuit Department working on transmitter- and receiver-based integrated photonic circuits. Since 2013, he has been in charge of Opto-Electronic Integrated Circuits OEIC Department.
Xing-Zhi Qiu (M'98) received the Ph.D. degree in applied sciences, electronics from Ghent University, Ghent, Belgium, in 1993. She joined the Department of information technology (INTEC) of Ghent University in 1986. She is currently a Group Leader of INTEC_design laboratory, an associated laboratory of IMEC, Belgium, and a Professor at INTEC Department of Ghent University. She gained 26 years R\&D experience within INTEC_design laboratory in the field of high speed $\mathrm{O} / \mathrm{E} / \mathrm{O}$ front-ends and physical layer hardware design for broadband optical networks in general and burst-mode receiver/transmitter technologies for passive optical networks in particular She is author or co-author of more than 150 publications and six patents on ASIC and telecom system designs.

Johan Bauwelinck (M'02) was born in Sint-Niklaas, Belgium, in 1977. He received the engineering degree in applied electronics and the Ph.D. degree in applied sciences, electronics from Ghent University, Ghent, Belgium, in 2000 and 2005, respectively. He has been a Research Assistant in the INTEC_design laboratory, Ghent University, since 2000, and he is currently a full-Time Tenure Track Professor. His research focuses on high-speed, high-frequency (opto-) electronic circuits and systems and he is a member of the ECOC technical program committee.

Xin Yin (M'06) was born in Chongqing, China, in 1977. He received the B.E. and M.Sc. degrees in electronics engineering from the Fudan University, Shanghai, China, in 1999 and 2002, respectively, and the Ph.D. degree in applied sciences, electronics from Ghent University, Ghent, Belgium, in 2009. Since 2007, he has been a Researcher in IMEC-INTEC/Ghent University. He is also collaborating in European and international projects such as DISCUS, Phoxtrot, MIRAGE and GreenTouch consortium. His current research interests include high-speed opto-electronic circuits and subsystems, with emphasis on burst-mode receiver and CDR/EDC for optical access networks, and lowpower mixed-signal integrated circuit design for telecommunication applications. He is author or co-author of more than 50 national and international publications, both in journals and in proceedings of conferences. 